\title{
Investigations of Interferon-Lambda for the Treatment of Cancer
}

\author{
Andrew Stiffa ${ }^{a}$ William Carson 3 rd $^{\text {b }}$ \\ ${ }^{a}$ Biomedical Sciences Graduate Program and ${ }^{b}$ Division of Surgical Oncology, The Ohio State University, \\ Columbus, Ohio, USA
}

\section{Key Words}

Interferon-lambda - NK cells · Cancer · Apoptosis .

Cytokines $\cdot$ Immune response

\begin{abstract}
Interferon-lambda (IFN- $\lambda$ ), a recently discovered cytokine, overlaps broadly with type I IFN signaling, producing antiviral, antiproliferative, and proapoptotic responses. In comparison to type I IFNs, IFN- $\lambda$ has a limited spectrum of responsive tissues due to variation in expression of the IFN- $\lambda$ receptor IFNLR1. Type I IFNs have been investigated for their antitumor effects and used in the clinical setting for a number of different cancers. Given the overlap in signaling and function between IFN- $\lambda$ and type I IFNs, IFN- $\lambda$ has also drawn interest for the treatment of cancer. To date, a number of studies using both murine and human models of cancer have investigated the antitumor effects of IFN- $\lambda$. These studies have found that IFN- $\lambda$ is capable of directly targeting cancer cells to reduce their tumorigenicity, induce cell cycle arrest, and cause apoptosis. In addition, IFN- $\lambda$ has been shown to have indirect effects against cancer cells through immune system responses and immune modulatory effects. This review aims to detail the findings of studies investigating IFN- $\lambda$ for the treatment of cancer as well as suggest areas of potential interest for future studies.

(c) 2015 S. Karger AG, Basel
\end{abstract}

Both authors were equal contributors.

\section{Introduction}

While the scientific community has been aware of interferon (IFN) proteins for over 50 years, type III IFNs or IFN- $\lambda$ were first identified roughly 12 years ago. The IFN- $\lambda$ lambda family in humans has traditionally consisted of three members: IFN- $\lambda 1$ (IL-29), IFN- $\lambda 2$ (IL$28 \mathrm{~A}$ ), and IFN- $\lambda 3$ (IL-28B) whose genes are all clustered in the q13.13 region of chromosome $19[1,2]$. These new proteins are related to type I IFN and IL-10 family members $[3,4]$. The murine IFN- $\lambda$ gene cluster is located on chromosome $7 \mathrm{~A} 3$ and also consists of three genes, but only the IFN- $\lambda 2$ and IFN- $\lambda 3$ genes encode functional proteins, whereas the IFN- $\lambda 1$ gene has lost exon 2 and acquired a stop codon in exon 1 [5]. In addition, a new IFN- $\lambda$ family member, IFN $-\lambda 4$, was recently discovered in humans upstream of the gene encoding IFN- $\lambda$ 3. IFN- $\lambda 4$ was found to be similar to IFN- $\lambda 3$ and its expression was associated with lower levels of hepatitis $\mathrm{C}$ virus RNA prior to treatment, but impaired response to treatment with pegylated IFN- $\alpha$ and ribavirin. Several studies have also identified several polymorphisms within the IFN- $\lambda$ gene locus of humans, particularly near the IL-28B gene, that have prognostic value in the setting of hepatitis infection $[6,7]$. While IFN- $\lambda$ family members are highly similar to each other in their amino acid sequences, they are only approximately 12 and 15\% identical to type I IFNs and IL-10 family members, respectively $[1,2,8]$. Consistent with this, IFN- $\lambda$ has been found to interact with and sig-

Dr. Andrew Stiff

Biomedical Sciences Graduate Program

The Ohio State University

Columbus, OH 43221 (USA)

E-Mail Andrew.Stiff@osumc.edu 
nal through a unique heterodimeric receptor complex consisting of IFN- $\lambda$ receptor 1 (IFNLR1) and the IL-10 receptor subunit 2 (IL-10R2) [1-4]. Despite binding to a distinct receptor, IFN- $\lambda$ has antiviral functions and downstream signaling pathways that overlap significantly with type I IFN $[1,2,9,10]$. After binding to their respective receptor complexes, both IFN- $\lambda$ and type I IFNs activate Jak1 and Tyk2 kinases, which phosphorylate STAT1 and STAT2 on tyrosine residues. This leads to STAT1 and STAT2 association with IFN regulatory factor 9 forming the IFN-stimulated gene factor 3 transcriptional complex and the expression of IFN-stimulated genes. IFN-stimulated gene induction by type I IFNs and IFN- $\lambda$ results in antiviral, antiproliferative, and proapoptotic functions [1, $2,11,12]$. While these responses are similar, it has been noted that IFN- $\lambda$ stimulation causes a prolonged activation of STAT1 and STAT2 as well as a delayed but stronger induction of IFN-stimulated genes compared to type I IFN $[1,2,13]$. In the setting of cancer, IFN- $\lambda$ has been shown to exert direct effects against cancer cells by promoting cell cycle arrest and apoptosis as well as indirect effects through modulation of immune responses [14].

\section{IF $N-\lambda$ Tissue Specificity}

Despite the overlapping signaling pathways and antiviral functions performed by IFN- $\lambda$ and type I IFNs, several important differences have emerged. While the type I IFN receptors IFNAR1 and IFNAR2 are constitutively expressed in the human body, expression of the IFN- $\lambda$ receptor subunit IFNLR1 is much more restricted $[1,2]$. In an analysis of the expression pattern of IFNLR1 in human tissues, it was found that IFNLR1 mRNA levels were highest in the lung, heart, liver, and prostate, while low mRNA levels were detected in the central nervous system, bone marrow, testis, uterus, and skeletal muscle $[1,2,15]$. Within the skin, keratinocytes and melanocytes showed high expression of IFNLR 1 and response to IFN- $\lambda$, while dermal fibroblasts, endothelial cells and adipocytes did not express IFNLR1 and were unresponsive to even high doses. High levels of IFNLR1 and responsiveness to IFN- $\lambda$ have also been detected in human hepatocytes $[1,2,15$, 16]. Studies in mice have shown a relatively similar pattern of IFN- $\lambda$ responsiveness in different tissues, with the stomach, intestines, and lungs being the most responsive, while the central nervous system and spleen are relatively insensitive. In particular, it was found that the epithelial cells of most tissues express IFNLR1 and are responsive to IFN- $\lambda$ when tested in mice $[1,2,17,18]$.

\section{IFN- $\lambda$ and Immune Cell Responses}

The relationship between IFNLR1 expression and IFN- $\lambda$ responsiveness is not as straightforward in immune cell subsets. Witte et al. [15] tested the responsiveness of T cells, B cells, monocytes, and NK cells to doses of IFN- $\lambda$ as high as $1 \mu \mathrm{g} / \mathrm{ml}$ and could detect no response in terms of phosphorylation of STAT1 and STAT3 or increased MHC I expression. The addition of factors known to stimulate these immune cell populations prior to IFN- $\lambda$ treatment made no difference. While it was found that naïve human $\mathrm{B}$ and $\mathrm{T}$ cells expressed significant amounts of IFNLR1, it was determined that they were essentially unresponsive to IFN- $\lambda$ in terms of STAT1 and STAT3 activation or upregulation of MHC-I expression [1, 2, 15]. It was originally suggested that this discrepancy could be explained by the expression of an IFNLR1 splice variant (sIFNLR1) lacking the transmembrane domain. This splice variant was thought to be a soluble form of the receptor that could function as a decoy receptor, thereby negatively regulating IFN $-\lambda$ signaling in lymphoid tissue $[1,4,15]$. This hypothesis was tested by transiently overexpressing sIFNLR1 in HepG2 cells. It was found that sIFNLR1 was secreted into the culture media and could bind IFN- $\lambda$, thereby inhibiting its ability to induce signaling $[1,15]$. In addition, human monocytes and NK cells have also been found to express IFNLR1, but are unresponsive to IFN- $\lambda[1,2,15,19]$. However, conflicting reports have emerged which show that monocytes and $\mathrm{T}$ cells are capable of responding to IFN- $\lambda$ with altered cytokine production. It has been reported that despite a lack of STAT activation or MHC-I expression, monocytes and macrophages do respond to IFN- $\lambda$ by producing IL- 6 , IL8 , and IL-10 [20]. In addition, IFN- $\lambda$ has been found to decrease IL- 5 and IL- 13 production by naïve and memory human $\mathrm{T}$ cells while also increasing production of IFN- $\gamma[20,21]$. This suggests the existence of a STATindependent signaling network that regulates cytokine production in response to IFN- $\lambda$ in these cells $[1,2]$.

\section{Mechanism of IFNLR1 Expression}

A recent report investigated the mechanism for tissue specific expression of IFNLR1 by comparing IFNLR1 expressing hepatocyte-derived cell lines Huh7 and HepG2 to brain glia-derived cell lines U87 and U373 that do not express IFNLR1 [22]. These authors found that CpG islands in the promoter region of the IFNLR1 gene were hypermethylated in the U87 cell line compared to Huh7. 
However, treatment of U87 cells with the hypomethylating agent decitabine produced only a minor increase in IFNLR1 mRNA expression, indicating that higher-order chromatin modifications likely also influence IFNLR1 expression. In line with this, they found that Huh7 cells were enriched for markers of open and transcriptionally active chromatin within the IFNLR1 promoter, while U87 cells were enriched for markers of closed and transcriptionally inactive chromatin. Furthermore, treatment of U87 cells with nonspecific histone deacetylase inhibitors (HDACi) and decitabine resulted in significantly increased expression of IFNLR1 in U87 cells as well as cell lines from a number of other tissue types, indicating the response was not tissue specific. Importantly, IFN- $\lambda$ treatment of U87 cells pretreated with HDACi and decitabine to increase IFNLR1 expression showed increased responsiveness to IFN- $\lambda$ evidenced by STAT1 phosphorylation, IFN-stimulated gene expression, and antiviral protection. The combination of HDACi and IFN- $\lambda$ treatment of the U87 glioblastoma and murine B16 melanoma cell line suppressed proliferation and induced apoptosis in these cell lines to a greater extent than either agent alone. These findings suggest that inducing expression of IFNLR1 can be a means to sensitize cells to the anticancer effects of IFN- $\lambda$, although other mechanisms of interaction cannot be ruled out given the nonspecific action of HDACi.

\section{IFN and Cancer Treatment}

IFN- $\alpha$ has been extensively studied as an anticancer therapeutic. It has been shown to have antiproliferative, proapoptotic, and immunomodulatory properties in a variety of cancer systems [1, 23-26]. Furthermore, IFN- $\alpha$ has been used extensively in the clinic to treat several malignancies including melanoma, renal cell carcinoma, chronic myeloid leukemia, Kaposi's sarcoma, and multiple myeloma, among others $[2,23]$. In the setting of melanoma, adjuvant therapy IFN- $\alpha$ has produced significant improvements in relapse-free and overall survival in large randomized trials $[27,28]$. However, these beneficial effects were only obtained with very high doses $\left(20 \mathrm{IU} / \mathrm{m}^{2}\right.$ i.v. 5 doses/week), while studies examining the effects of low-dose IFN- $\alpha$ failed to produce major effects $[29,30]$. Such high doses are typically associated with significant side effects such as myelosuppression, neurotoxicity, fever, chills, nausea, and joint pain that sometimes forces the discontinuation of the therapy or lowering of the dose. In many instances these complications reduce the

IFN- $\lambda$ for the Treatment of Cancer clinical effectiveness of IFN- $\alpha$ therapy, and they are thought to be related to the ubiquitous expression of type I IFN receptors in the human body and their strong effect on immune cells. Given that IFN- $\lambda$ has significant overlap with type I IFNs in terms of signaling, antiproliferative, and proapoptotic functions, investigations have been initiated into its potential as a new cancer therapy. In addition, given that the profile of IFN $-\lambda$ responsive cell types is much more restricted compared to IFN- $\alpha$, it has been hypothesized that IFN- $\lambda$ could mediate the beneficial aspects of IFN- $\alpha$ with fewer side effects $[1,2]$.

\section{Antitumor Effects of IFN- $\lambda$}

\section{Initial Investigation of Anticancer Properties of IFN- $\lambda$} in Murine Models

Given the clinical use of IFN- $\alpha$ for the treatment of melanoma, the potential to use IFN- $\lambda$ as a less toxic alternative was pursued. In an initial investigation, Lasfar et al. [5] showed that murine B16 melanoma cells responded to IFN- $\lambda$ with STAT activation, upregulation of MHC I, and antiviral protection. Subsequently, they transfected $B 16$ cells with a plasmid encoding murine IFN- $\lambda$ to establish B16 cells that constitutively expressed and secreted murine IFN- $\lambda$ (B16-IFN- $\lambda$ ). Interestingly, they detected no difference in the proliferation rate between parental B16 cells, control vector-transfected B16 cells, or B16IFN- $\lambda$ cells in vitro. They then investigated the potential of constitutive IFN $-\lambda$ production to affect tumorigenicity in vivo using C57BL/6 mice. In comparison to parental $\mathrm{B} 16$ or vector-transfected $\mathrm{B} 16$ cells that formed tumors in all mice within 20 days, B16-IFN- $\lambda$ cells produced tumors significantly later or not at all. In addition, when B16IFN- $\lambda$ cells were mixed with equal amounts of B16 parental cells and injected into mice, tumor development was significantly delayed again with a number of mice never forming tumors. To investigate if the decreased tumorigenicity of B16-IFN- $\lambda$ cells was due to direct effects of IFN- $\lambda$ on B16 cells or indirect mechanisms such as immune system activation, B16 cells expressing but unresponsive to IFN- $\lambda$ due to lack of IFNLR1 (B16-IFN- $\lambda$ res) were generated. Similar to B16-IFN- $\lambda$ cells, B16-IFN- $\lambda$ res cells also demonstrated reduced tumorigenicity, indicating an indirect mechanism likely explained by the effect of IFN- $\lambda$. Histological examination showed that B16 parental tumors had enhanced vascularity and a higher mitotic rate than B16-IFN- $\lambda$ tumors, suggesting a role for IFN- $\lambda$ in the tumor microenvironment. This group also reported that keratinocytes, which are known to interact 
with melanocytes, were highly responsive to IFN- $\lambda$. The authors suggested that the response of keratinocytes and other stromal cells in the tumor microenvironment to IFN- $\lambda$ could play a role in inhibiting tumor growth.

In a subsequent study, Sato et al. [31] generated IFN$\lambda$-expressing murine B16F0 cells (B16F0-IFN- $\lambda$ ). These B16F0-IFN- $\lambda$ cells showed reduced soft agar colony formation and proliferation in vitro. The authors further demonstrated that B16F0-IFN- $\lambda$ cells had increased expression of $\mathrm{p} 21$ and decreased phosphorylation of $\mathrm{Rb}$, indicating cell cycle arrest. B16F0-IFN- $\lambda$ cells also showed increased activation of caspase 3 and 7 in vitro, indicating increased apoptosis due to IFN- $\lambda$ exposure. In addition, B16F0-IFN- $\lambda$ cells showed reduced pulmonary metastasis following tail-vein injection. This reduction of metastasis was associated with increased cellular infiltration of the lungs, indicating a potential immune response the malignant cells. In the presence of IFN- $\lambda$, depletion of $\mathrm{CD} 4+$ and CD8+ T cells did not result in increased B16F0IFN- $\lambda$ tumor growth; however, NK cell depletion led to progressive tumor growth, suggesting that NK cells could be the predominant cell type involved in the antitumor action of IFN- $\lambda$. A study by Numasaki et al. [32] using a murine fibrosarcoma model provided additional support for an indirect antitumor mechanism of IFN- $\lambda$. This study showed that IFN- $\lambda$-expressing murine MCA205 cells showed decreased tumor growth and pulmonary metastasis, corroborating previous work. They also showed that sublethal irradiation of the bone marrow was able to abolish this effect, supporting the importance of an immune cell subset in mediating this effect. They went on to show that antibody depletion of NK cells, CD8 T cells, and neutrophils reduced the effects of IFN- $\lambda$, strengthening the argument for an indirect immune-mediated effect.

Oncolytic Virus Induction of IFN- $\lambda$ Promotes NK Cell Targeting of Murine Melanoma

In a study investigating the oncolytic virus vesicular stomatitis virus (VSV), Wongthida et al. [33] hypothesized its mechanism of action against cancer cells in vivo was at least in part mediated by activation of immune cells by the virus that subsequently could target tumor cells. They initially observed that coculture of B16ova cells with bone marrow cells from $\mathrm{C} 57 \mathrm{Bl} / 6$ mice alone produce no cytotoxic effects, but B16ova cells were killed when VSV was added to this coculture. This effect occurred with or without the addition of VSV neutralizing serum, which blocks infection and direct oncolysis of B16ova cells by VSV, indicating the virus activated immune cells within the bone marrow cell population that were likely killing the tumor cells. They subsequently found that a B16-derived cell line generated in their lab (B16LIF) was insensitive to VSV-activated BM cells in vitro and in vivo compared to the highly sensitive B16ova in both settings. They found B16ova cells overexpressed IFNLR1 23-fold compared to B16LIF cells, and that B16LIF did not express IFNLR1 even after VSV infection. They went on to show that antibody-mediated blockade of IFN- $\lambda$ signaling abolished VSV-activated bone marrow cell killing of B16ova cells, suggesting IFN- $\lambda$ was critical for this function. They were also able to show that depletion of macrophages and Gr1+ cells from bone marrow cell cultures significantly reduced the amount of IFN- $\lambda$ produced in response to VSV with or without B16ova cells. Depletion of GR1+ cells and macrophages from bone marrow B16ova cell cocultures also significantly reduced the cytotoxic effect produced by VSV treatment, while depletion of other immune cell subsets had no effect. This study also found that while NK cells were not an important source of IFN- $\lambda$ in response to VSV, their depletion from B16ova bone marrow cell cocultures reduced the killing of B16ova cells to a similar degree as IFNLR1 blockade. In search of an explanation for this phenomenon Wongthida et al. found treatment of B16ova cells with IFN- $\lambda$ increased their expression of several NK cell stimulatory ligands, while this did not occur in IFNLR1deficient B16LIF cells. Furthermore, IFN- $\lambda$ treatment of B16ova cells was able to induce IFN- $\gamma$ production by NK cells. These in vitro findings were corroborated in vivo when NK cell depletion significantly reduced the effectiveness of VSV treatment of B16ova tumors. Finally, these researchers used a lentivirus vector to induce expression of IFNLR1 in B16LIF cells, which normally do not express IFNLR1 and are insensitive to VSV-activated bone marrow cell killing or VSV treatment in vivo. Following expression of IFNLR1, B16LIF cells were as sensitive as B16ova cells to VSV treatment in vitro and in vivo, and could effectively activate NK cells following IFN- $\lambda$ treatment. From this evidence the authors concluded that IFN- $\lambda$ produced by GR $1+$ cells and macrophages in response to VSV treatment led to increased recognition and killing of murine melanoma cells by NK cells. Abushahba et al. [34] corroborated these findings using a murine model in which BNL hepatoma cells were engineered to express IFN $-\lambda$. These authors found that while NK cells were unable to directly respond to IFN- $\lambda$, tumors expressing IFN- $\lambda$ showed increased NK cell infiltration, and depletion of NK cells from splenocytes reduced their cytotoxic activity.
246

J Innate Immun 2015;7:243-250 DOI: $10.1159 / 000370113$
Stiff/Carson III 


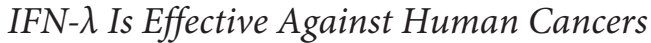

Work done by our group aimed at extending these findings in murine models of melanoma into human systems. As an initial step, mRNA expression of the components of the IFN- $\lambda$ receptor complex IFNLR1 and IL10R2 were assessed in eight human melanoma cell lines by RT-PCR. The eight cell lines showed equivalent expression of IL-10R2, but expression of IFNLR1 was variable with $1174 \mathrm{Mel}$ showing undetectable levels. All cell lines tested except $1174 \mathrm{Mel}$ were shown to be responsive to IFN- $\lambda$ by induction of STAT 1 and STAT 2 phosphorylation. Interestingly, IFN- $\lambda$ treatment resulted in STAT5 phosphorylation in $1174 \mathrm{Mel}$ despite it apparently lacking IFNLR1 expression. It was hypothesized that this could occur through IFN- $\lambda$ 's interaction with other cytokine receptors such as IL-10R1 or IL-20R1. In addition, it was found that IFN- $\lambda$ treatment did not cause activation of the AKT, ERK, or SAPK/JNK pathways. Microarray gene expression analysis on the $1106 \mathrm{Mel}$ cell line following stimulation with IFN- $\lambda$ revealed that IFN-stimulated genes such as IFI27, RSAD2, and IFI44L were the most commonly induced genes. IFN-stimulated gene expression was also shown to be dose and time dependent. The potential antitumor mechanisms of IFN- $\lambda$ against human melanoma cell lines were investigated next. The potential of IFN- $\lambda$ to prime NK cells and enhance their ability to lyse target melanoma cells was tested. While NK cells were found to express both IL-10R2 and IFNLR1, and showed activation of Jak-STAT signaling in response to IFN- $\lambda$, no enhancement of NK cell cytotoxicity was found. The lack of NK cell activity in these experiments was surprising given the evidence supporting the importance of NK cells in murine models. However, these experiments were not conducted in the context of additional immune cells such as macrophages and other lymphocytes, which could play an important role in the murine models. The potential for IFN- $\lambda$ to exert direct effects against melanoma cells lines was examined next. It was found that IFN- $\lambda$ treatment had no effect on the proliferation of melanoma cell lines, but that the F01 cell line showed a dose-dependent increase in apoptosis following 48 -hour treatment with IFN- $\lambda$. It was then shown that the combination of IFN- $\lambda$ and the proteasome inhibitor bortezomib or IFN- $\lambda$ in combination with temozolomide was capable of producing synergistic apoptosis in F01 melanoma cells. Finally, the expression of IFN- $\lambda$ receptor components in benign nevi and primary melanoma lesions was evaluated. All benign nevi examined lacked both IL-10R2 and IFNLR1, while 6 of 8 primary melanoma lesions expressed both components [19].

IFN- $\lambda$ for the Treatment of Cancer
Other groups have also explored the effects of IFN- $\lambda$ on a number of different human cancers including bladder carcinoma, Burkitt's lymphoma, colorectal carcinoma, glioblastoma, non-small cell lung cancer, esophageal carcinoma, and osteosarcoma, among others $[1,2]$. In general, these studies have found that cell lines from these various cancers display a range of sensitivity to IFN- $\lambda$ treatment. This variability generally matches the expression of IFNLR1 by these cell lines, indicating its importance for the effectiveness of IFN- $\lambda$ treatment of cancer [35-38]. Recently, a report showed that IFN- $\lambda$ was expressed at higher levels in cervical samples from women infected with low-risk human papilloma virus (HPV) strains compared to high-risk HPV strains. In addition, IFN- $\lambda$ levels decreased significantly with abnormal cytology, suggesting high-risk HPV strains may be able to counteract the IFN- $\lambda$ response and that the loss of IFN- $\lambda$ expression might be related to disease progression [39]. Many of these studies have shown IFN- $\lambda$ is capable of producing cell cycle arrest in cancer cells through induction of p21 and decreased phosphorylation of $\mathrm{Rb}$ as well and the induction of apoptosis evidenced by caspase $3 / 7$ activation or PARP cleavage [31, 36, 37, 40-42]. Several studies have also shown that IFN- $\lambda$ can increase the effectiveness of several widely used chemotherapeutic agents, suggesting its potential to be used as an adjuvant to existing chemotherapy regimens $[19,36,37]$.

\section{Immune Modulatory Effects of IFN- $\lambda$}

Reports investigating the immune modulatory effects of IFN- $\lambda$ have shown that it is capable of influencing the balance between Th1 and Th2 immune responses [20, 21]. Dai et al. [21] showed that stimulation of human PBMCs with anti-CD2/3/28 beads in the presence of IFN- $\lambda$ led to a decrease in the production of the Th2 cytokine IL-13 and an increase in IFN- $\gamma$ production. The authors further showed that IFN- $\lambda$ led to an immediate decrease in IL-13 production, and it was only at later time points that IFN- $\gamma$ increased. They concluded from this that IFN- $\lambda$ acted primarily to inhibit IL-13 production and the increased IFN- $\gamma$ was a consequence of decreased Th2 cytokine production. They next evaluated the effect of IFN- $\lambda$ on the production of Th2 cytokines IL-13 and IL- 5 by naïve and memory T cells stimulated with antiCD2/3/28 beads in the presence of IL- 4 , which promotes the development of a Th2 response. The addition of IL- 4 led to a significant increase in IL-13 and IL-5 production by naïve $\mathrm{T}$ cells, but did not further increase the produc-

J Innate Immun 2015;7:243-250

DOI: $10.1159 / 000370113$ 
tion of these cytokines by memory T cells, indicating IL-4 had a selective effect on naïve T cells. For both memory and naïve $T$ cells, the addition of IFN- $\lambda$ led to reduced production of IL-13 and IL-5. They were also able to show that activation of naïve $\mathrm{T}$ cells led to an increase in their expression of the IL- 4 receptor (IL-4Ra) while this did not occur in memory $\mathrm{T}$ cells. Furthermore, the presence of IFN- $\lambda$ was able to block the induction of the IL- 4 receptor on naïve T cells after their activation. Finally, it was found that the addition of IFN- $\lambda$ to naïve T cells activated with anti-CD2/3/28 beads in the presence of IL- 4 led to a significant decrease in expression of the Th2 master transcription factor GATA3, while the same treatments produced only a slight decrease in GATA3 expression by memory $\mathrm{T}$ cells. These data led the authors to conclude that IFN- $\lambda$ was capable of shutting down key pathways involved in Th2 development and therefore allowed the Th1 response to be more robust than would otherwise have occurred in the absence of IFN- $\lambda$. It should be noted that the ability of IFN- $\lambda$ to influence the development of Th1 and Th2 responses stands in contrast to the findings of Witte et al. [15] who found that T cells were unresponsive to IFN- $\lambda$ treatment. It has been shown that IFN- $\lambda$ can alter monocyte-derived dendritic cell development such that they diminish Th2 development, suggesting this disagreement could be caused by the effect of IFN- $\lambda$ on additional immune cell subsets $[15,20]$.

The potential for IFN- $\lambda$ 's influence on the balance between Th1 and Th2 immune responses to impact cancer was recently highlighted by an investigation of the IFNinducible gene USP18 in a mouse model of spontaneous breast cancer by Burkart et al. [43]. USP18 is known to be a negative regulator of type I IFN responses, and USP18 deficiency has been reported to have an inhibitory effect on the development of leukemia. This study used a mouse that was a cross between the polyomavirus middle $\mathrm{T}$ $(\mathrm{PyVmT})$ mouse model for breast cancer with a USP18 knockout (KO) mouse to generate PyVmT/USP18 KO mice. In comparison to PyVmT/USP18 WT mice, the PyVmT/USP18 KO mice showed no difference in tumor latency, but did demonstrate a significant improvement in survival and tumor burden. This effect was shown to not be the result of intrinsic differences in proliferation or apoptosis between USP18 KO and wild-type (WT) mammary epithelial cells in this model. Analysis of single-cell suspensions produced from the tumors of $\mathrm{PyVmT/USP} 18 \mathrm{KO}$ and WT mice revealed a significant increase in the number of CD4+ T cells in tumors from USP18 KO mice. There was also an observed increase in CD8+ T cells, NK cells, and macrophages that did not reach statistical significance, while there was no difference in myeloid-derived suppressor cells. Importantly, depletion of CD4+ T cells from FVB WT mice injected with PyVmT/USP18 KO mammary epithelial cells showed enhanced tumor growth, highlighting that reduced tumor growth of USP18 KO tumors was dependent on CD4+ T cells. Interestingly, depletion of CD4+ T cells from FVB WT mice that were injected with both $\mathrm{PyVmT/USP} 18 \mathrm{KO}$ and USP18 WT cells produced a protective effect, indicating USP18 was potentially regulating the pro- and antitumor effects of CD4+ T cells.

To investigate this, Burkart et al. [43] analyzed the expression of T cell-specific chemokines and found that the Cxcr3 ligands Cxcl10 and Cxcl11 were significantly upregulated in PyVmT/USP18 KO tumors. Since expression of Cxcr3 is associated with Th1 cells, the authors investigated if PyVmT/USP18 KO tumors showed a Th1 cytokine profile and found they had increased expression of IFN- $\gamma$ and decreased expression of the Th2 cytokines IL-4 and IL-13. In addition, when PyVmT/USP18 WT cells were injected into USP18 KO and WT mice, no difference in tumor growth was observed, indicating that it was specifically the lack of USP18 expression in tumor cells that was responsible for this effect. PyVmT/USP18 $\mathrm{KO}$ cells stably expressing Cxcl10 shRNA showed increased tumor growth and decreased CD4+ T cell infiltration, highlighting the importance of increased Cxcl10 expression by USP18 KO tumors.

Subsequently, USP18 was identified to be a negative regulator of IFN- $\lambda$ signaling and Cxcl10 expression in cells with rescued USP18 expression. Finally, injection of PyVmT/USP18 KO mammary epithelial cells with reduced IFNLR1 expression showed increased tumor growth. This work highlights that in the setting of hypersensitivity to IFN- $\lambda$ signaling due to loss of USP18, the cytokine milieu of the tumor microenvironment is skewed towards a Th1 response that results in reduced tumor growth [43].

\section{Conclusion and Future Directions}

IFN- $\lambda$ is a recently discovered cytokine that overlaps significantly with type I IFN in terms of signaling pathways, but differs substantially from type I IFN in terms of tissue responsiveness. This is due to variation in IFNLR1 expression between tissue types, which appears to be epigenetically regulated, potentially allowing for drugs such as HDAC inhibitors to increase cell types that can respond to IFN- $\lambda$. IFN- $\lambda$ has also been shown to exert anti- 
tumor effects in both murine and human models. This has been shown to occur through direct effects on target tumor cells as well through indirect immune-mediated responses. IFN- $\lambda$ can also potentially alter the balance between Th1 and Th2 immune responses, which may be able to be taken advantage of for the treatment of cancer.

Increasing evidence suggests that dysfunction of the immune system plays a critical role in the progression of human malignancies [44]. In fact, some have suggested that evasion of the immune system is in fact a hallmark of cancer [45]. A number of different immune cell subsets including T cells, B cells, NK cells, dendritic cells, macrophages, and myeloid-derived suppressor cells have been identified within the stroma of the tumor microenvironment. These immune cells, as thoroughly reviewed elsewhere [44], are frequently located at distinct locations within the tumor microenvironment, suggesting different functions in controlling or promoting tumor growth. Studies have also shown that an increased lymphocytic infiltrate is associated with an improved prognosis [44].

The ability of IFN- $\lambda$ to inhibit the development of potentially protumor Th2 immune responses in favor of antitumor Th1 responses suggests it would be interesting to evaluate the efficacy of IFN- $\lambda$ in combination with emerging immunotherapeutic approaches. In particular, investigation of the ability of IFN- $\lambda$ to increase expression of potent $\mathrm{T}$ cell chemoattractant $\mathrm{Ccr} 3$ ligands by human cancers as it does in the murine model discussed above could be productive. This would allow for investigation of the ability of IFN- $\lambda$ to promote the recruitment of T cells into the tumor microenvironment, and open the door for the investigation of IFN- $\lambda$ as a complimentary therapy to the new immune checkpoint inhibitors such as ipilimumab (anti-CTLA4) or antibodies directed against PD-1/PD-L1. These immune checkpoint inhibitors function to block inhibitory receptors expressed on $\mathrm{T}$ cells whose ligands are often found within the tumor microenvironment or are directly expressed by tumor cells and cause loss of T cell effector function $[46,47]$. A common side effect of these checkpoint inhibitors is the development of adverse autoimmune reactions, which suggests their combination with other immune modulatory agents or cytokines could be dangerous. However, as noted above, the limited expression of the IFNLR1 and its lower side-effect profile relative to IFN- $\alpha$ could make IFN- $\lambda$ a safer alternative in this setting.

\section{References}

- 1 Lasfar A, Abushahba W, Balan M, CohenSolal KA: Interferon lambda: a new sword in cancer immunotherapy. Clin Dev Immunol 2011;2011:349575.

2 Steen HC, Gamero AM: Interferon-lambda as a potential therapeutic agent in cancer treatment. J Interferon Cytokine Res 2010;30:597602

3 Kotenko SV, Gallagher G, Baurin VV, LewisAntes A, Shen M, Shah NK, Langer JA, Sheikh F, Dickensheets H, Donnelly RP: IFN-lambdas mediate antiviral protection through a distinct class II cytokine receptor complex. Nat Immunol 2003;4:69-77.

4 Sheppard P, Kindsvogel W, Xu W, Henderson K, Schlutsmeyer S, Whitmore TE, Kuestner R, Garrigues U, Birks C, Roraback J, Ostrander C, Dong D, Shin J, Presnell S, Fox B, Haldeman B, Cooper E, Taft D, Gilbert T, Grant FJ, Tackett M, Krivan W, McKnight G, Clegg C, Foster D, Klucher KM: IL-28, IL-29 and their class II cytokine receptor IL-28R. Nat Immunol 2003;4:63-68.

5 Lasfar A, Lewis-Antes A, Smirnov SV, Anantha S, Abushahba W, Tian B, Reuhl K, Dick ensheets H, Sheikh F, Donnelly RP, Raveche E, Kotenko SV: Characterization of the mouse IFN-lambda ligand-receptor system: IFNlambdas exhibit antitumor activity against B16 melanoma. Cancer Res 2006;66:44684477.
6 Boglione L, Cusato J, Allegra S, Esposito I Patti F, Cariti G, Di Perri G, D’Avolio A: Role of IL28-B polymorphisms in the treatment of chronic hepatitis B HBeAg-negative patients with peginterferon. Antiviral Res 2014;102: 35-43.

7 Jimenez-Sousa MA, Fernandez-Rodriguez A, Guzman-Fulgencio M, Garcia-Alvarez M, Resino S: Meta-analysis: implications of interleukin-28B polymorphisms in spontaneous and treatment-related clearance for patients with hepatitis C. BMC Med 2013; 11:6.

8 Gad HH, Dellgren C, Hamming OJ, Vends S, Paludan SR, Hartmann R: Interferon-lambda is functionally an interferon but structurally related to the interleukin-10 family. J Biol Chem 2009;284:20869-20875.

9 Ank N, West H, Bartholdy C, Eriksson K, Thomsen AR, Paludan SR: Lambda interfer on (IFN-lambda), a type III IFN, is induced by viruses and IFNs and displays potent antiviral activity against select virus infections in vivo. J Virol 2006;80:4501-4509.

10 Ank N, Iversen MB, Bartholdy C, Staeheli P, Hartmann R, Jensen UB, Dagnaes-Hansen F, Thomsen AR, Chen Z, Haugen H, Klucher K, Paludan SR: An important role for type III interferon (IFN-lambda/IL-28) in TLR-induced antiviral activity. J Immunol 2008;180: 2474-2485.
11 Dumoutier L, Lejeune D, Hor S, Fickenscher $\mathrm{H}$, Renauld JC: Cloning of a new type II cytokine receptor activating signal transducer and activator of transcription (STAT)1, STAT2 and STAT3. Biochem J 2003;370: 391-396.

12 Dumoutier L, Tounsi A, Michiels T, Sommereyns C, Kotenko SV, Renauld JC: Role of the interleukin (IL)-28 receptor tyrosine residues for antiviral and antiproliferative activity of IL-29/interferon-lambda 1: similarities with type I interferon signaling. J Biol Chem 2004;279:32269-32274.

13 Maher SG, Sheikh F, Scarzello AJ, RomeroWeaver AL, Baker DP, Donnelly RP, Gamero AM: IFNalpha and IFNlambda differ in their antiproliferative effects and duration of JAK/ STAT signaling activity. Cancer Biol Ther 2008;7:1109-1115

14 Lasfar A, Cohen-Solal KA: Emergence of IFN-lambda as a potential antitumor agent; in You Y (ed): Targets in Gene Therapy. 2011, InTech, DOI: 10.5772/18783.

15 Witte K, Gruetz G, Volk HD, Looman AC, Asadullah K, Sterry W, Sabat R, Wolk K: Despite IFN-lambda receptor expression, blood immune cells, but not keratinocytes or melanocytes, have an impaired response to type III interferons: implications for therapeutic applications of these cytokines. Genes Immun 2009;10:702-714. 
16 Wolk K, Witte K, Witte E, Proesch S, Schulze Tanzil G, Nasilowska K, Thilo J, Asadullah K, Sterry W, Volk HD, Sabat R: Maturing dendritic cells are an important source of IL-29 and IL-20 that may cooperatively increase the innate immunity of keratinocytes. J Leukoc Biol 2008;83:1181-1193.

- 17 Sommereyns C, Paul S, Staeheli P, Michiels T: IFN-lambda (IFN- $\lambda$ ) is expressed in a tissuedependent fashion and primarily acts on epi thelial cells in vivo. PLoS Pathog 2008;4: e1000017.

- 18 Zhang L, Fok JJ, Mirabella F, Aronson LI, Fryer RA, Workman P, Morgan GJ, Davies FE: Hsp70 inhibition induces myeloma cell death via the intracellular accumulation of immu noglobulin and the generation of proteotoxic stress. Cancer Lett 2013;339:49-59.

19 Guenterberg KD, Grignol VP, Raig ET, Zimmerer JM, Chan AN, Blaskovits FM, Young GS, Nuovo GJ, Mundy BL, Lesinski GB, Carson WE 3rd: Interleukin-29 binds to melano ma cells inducing Jak-STAT signal transduction and apoptosis. Mol Cancer Ther 2010;9: 510-520.

20 Jordan WJ, Eskdale J, Srinivas S, Pekarek V, Kelner D, Rodia M, Gallagher G: Human interferon lambda-1 (IFN-lambda1/IL-29) modulates the Th1/Th2 response. Genes Immun 2007;8:254-261.

- 21 Dai J, Megjugorac NJ, Gallagher GE, Yu RY, Gallagher G: IFN-lambdal (IL-29) inhibit? GATA3 expression and suppresses Th2 responses in human naive and memory $\mathrm{T}$ cells. Blood 2009;113:5829-5838.

22 Ding S, Khoury-Hanold W, Iwasaki A, Robel MD: Epigenetic reprogramming of the type III interferon response potentiates antiviral activity and suppresses tumor growth. PLoS Biol 2014;12:e1001758.

23 Pasquali S, Mocellin S: The anticancer face o interferon alpha (IFN-alpha): from biology to clinical results, with a focus on melanoma. Curr Med Chem 2010;17:3327-3336.

24 Passalacqua R, Caminiti C, Buti S, Porta C, Camisa R, Braglia L, Tomasello G, Vaglio A, Labianca R, Rondini E, Sabbatini R, Nastasi G, Artioli F, Prati A, Potenzoni M, Pezzuol D, Oliva E, Alberici F, Buzio C; POLAR-01 Trial Investigators: Adjuvant low-dose interleukin-2 (IL-2) plus interferon-alpha (IFNalpha) in operable renal cell carcinoma (RCC): a phase III, randomized, multicentre trial of the Italian Oncology Group for Clini cal Research (GOIRC). J Immunother 2014; 37:440-447.

25 Khoo TL, Vangsted AJ, Joshua D, Gibson J: Interferon-alpha in the treatment of multiple myeloma. Curr Drug Targets 2011;12:437-446.

26 Stein BL, Tiu RV: Biological rationale and clinical use of interferon in the classical BCRABL-negative myeloproliferative neoplasms. J Interferon Cytokine Res 2013;33:145-153.
27 Kirkwood JM, Ibrahim JG, Sosman JA, Son dak VK, Agarwala SS, Ernstoff MS, Rao U: High-dose interferon alfa-2b significantly prolongs relapse-free and overall survival compared with the GM2-KLH/QS-21 vaccine in patients with resected stage IIB-III melanoma: results of intergroup trial E1694/ S9512/C509801. J Clin Oncol 2001;19:2370 2380.

28 Kirkwood JM, Strawderman MH, Ernstoff MS, Smith TJ, Borden EC, Blum RH: Interferon alfa- $2 \mathrm{~b}$ adjuvant therapy of high-risk resected cutaneous melanoma: the Eastern Cooperative Oncology Group Trial EST 1684. Clin Oncol 1996;14:7-17.

29 Cascinelli N, Belli F, MacKie RM, Santinami M, Bufalino R, Morabito A: Effect of longterm adjuvant therapy with interferon alpha$2 \mathrm{a}$ in patients with regional node metastases from cutaneous melanoma: a randomised tri al. Lancet 2001;358:866-869.

30 Kleeberg UR, Suciu S, Brocker EB, Ruiter DJ, Chartier C, Lienard D, Marsden J, Schadendorf D, Eggermont AM: Final results of the EORTC 18871/DKG 80-1 randomised phase III trial. rIFN-alpha2b versus rIFN-gamma versus ISCADOR $\mathrm{M}$ versus observation afte surgery in melanoma patients with either high-risk primary (thickness $>3 \mathrm{~mm}$ ) or regional lymph node metastasis. Eur J Cancer 2004;40:390-402.

31 Sato A, Ohtsuki M, Hata M, Kobayashi E, Mu rakami T: Antitumor activity of IFN-lambda in murine tumor models. J Immunol 2006; 176:7686-7694

32 Numasaki M, Tagawa M, Iwata F, Suzuki T, Nakamura A, Okada M, Iwakura Y, Aiba S, Yamaya M: IL-28 elicits antitumor responses against murine fibrosarcoma. J Immund 2007;178:5086-5098.

33 Wongthida P, Diaz RM, Galivo F, Kottke T, Thompson J, Pulido J, Pavelko K, Pease L, Melcher A, Vile R: Type III IFN interleu kin-28 mediates the antitumor efficacy of oncolytic virus VSV in immune-competent mouse models of cancer. Cancer Res 2010;70: 4539-4549.

34 Abushahba W, Balan M, Castaneda I, Yuan Y, Reuhl K, Raveche E, de la Torre A, Lasfar A, Kotenko SV: Antitumor activity of type I and type III interferons in BNL hepatoma model. Cancer Immunol Immunother 2010;59: 1059-1071.

35 Tezuka Y, Endo S, Matsui A, Sato A, Saito K, Semba K, Takahashi M, Murakami T: Potential anti-tumor effect of IFN- $\lambda 2$ (IL-28A) against human lung cancer cells. Lung Cance 2012;78:185-192.
36 Li Q, Kawamura K, Ma G, Iwata F, Numasaki M, Suzuki N, Shimada H, Tagawa M: Interferon-lambda induces G1 phase arrest or apoptosis in oesophageal carcinoma cells and produces anti-tumour effects in combination with anti-cancer agents. Eur J Cancer 2010;46: 180-190.

37 Fujie H, Tanaka T, Tagawa M, Kaijun N, Watanabe M, Suzuki T, Nakayama K, Numasaki M: Antitumor activity of type III interferon alone or in combination with type I interferon against human non-small cell lung cancer. Cancer Sci 2011;102:1977-1990.

38 Zitzmann K, Brand S, Baehs S, Göke B, Meinecke J, Spottl G, Meyer H, Auernhammer CJ: Novel interferon-lambdas induce antiproliferative effects in neuroendocrine tumor cells. Biochem Biophys Res Commun 2006;344: 1334-1341.

39 Cannella F, Scagnolari C, Selvaggi C, Stentella P, Recine N, Antonelli G, Pierangeli A: Interferon lambda 1 expression in cervical cells differs between low-risk and high-risk human papillomavirus-positive women. Med Microbiol Immunol 2014;203: 177-184.

40 Tagawa M, Kawamura K, Li Q, Tada Y, Hiroshima $\mathrm{K}$, Shimada $\mathrm{H}$ : A possible anticancer agent, type III interferon, activates cell death pathways and produces antitumor effects. Clin Dev Immunol 2011;2011:479013.

41 Li Q, Kawamura K, Okamoto S, Fujie H, Numasaki $M$, Namba M, Nagata $M$, Shimada $H$, Kobayashi H, Tagawa M: Adenoviruses-mediated transduction of human oesophageal carcinoma cells with the interferon-lambda genes produced anti-tumour effects. Br J Cancer 2011;105:1302-1312.

42 Li W, Lewis-Antes A, Huang J, Balan M, Kotenko SV: Regulation of apoptosis by type III interferons. Cell Prolif 2008;41: 960-979.

43 Burkart C, Arimoto K, Tang T, Cong X, Xiao N, Liu YC, Kotenko SV, Ellies LG, Zhang DE: Usp18 deficient mammary epithelial cells create an antitumour environment driven by hypersensitivity to IFN- $\lambda$ and elevated secretion of Cxcl10. EMBO Mol Med 2013;5:967982.

44 Fridman WH, Pages F, Sautes-Fridman C, Galon J: The immune contexture in human tumours: impact on clinical outcome. Nat Rev Cancer 2012;12:298-306.

45 Hanahan D, Weinberg RA: Hallmarks of cancer: the next generation. Cell 2011;144:646674.

46 Drake CG, Lipson EJ, Brahmer JR: Breathing new life into immunotherapy: review of melanoma, lung and kidney cancer. Nat Rev Clin Oncol 2014;11:24-37.

47 Pardoll DM: The blockade of immune checkpoints in cancer immunotherapy. Nat Rev Cancer 2012;12:252-264. 